\title{
CAPTURE AND HOLDING OF RESONANCE FAR FROM EQUILIBRIUM
}

\author{
L.A. KALYAKIN
}

\begin{abstract}
Capture into resonance occurs in nonlinear oscillating systems. The study of mathematical models of this phenomenon is a part of a modern theory of nonlinear oscillations. The known result in this field were obtained by averaging method in the asymptotic regime with a small parameter. In this way, an initial stage of the capture into resonance was studied in details.

The matter of this approach is an asymptotic passage to a model equation of mathematical pendulum kind. In the present work we consider an asymptotic construction at long time, which describes a slow evolution of a solution captured into resonance. The main aim is to determine a time interval, during which the resonance is held. The problem is reduced to studying a perturbation of a model equation of pendulum type. Our main success is the description of the time interval, in which the resonance is captured, and the description is given in terms of the data in the original problem. Formally we consider a nonlinear oscillating system with a small perturbation. The perturbation is described by an external pumping with a prescribed slowly changing frequency. For the solutions captured into the resonance, we consider asymptotics with respect to the small parameter. We write out an equation, the solution to which allows us to find the time of the capturing into resonance.
\end{abstract}

Keywords: nonlinear oscillations, perturbation, small parameter, asymptotics, capture in resonance.

Mathematics Subject Classification: 34E13

\section{INTRODUCTION}

1.1. Formulation of problem. The initial object is the system of two differential equation with a small parameter $0<\varepsilon \ll 1$ :

$$
\frac{d E}{d t}=\varepsilon F(E, S, \psi, \varepsilon t), \quad \frac{d S}{d t}=\Lambda(E, \varepsilon t)+\varepsilon G(E, S, \psi, \varepsilon t) .
$$

Here $\psi=\psi(t, \varepsilon)$ is a given function with a slowly varying derivative:

$$
\frac{d \psi(t, \varepsilon)}{d t}=\omega(\varepsilon t) \neq 0
$$

It is obvious that $\psi$ can be identified with the independent variable by making an appropriate change of a fast time $t$ and reducing the problem to the case $\omega=1$. Equations in form (1) are more convenient for applications and numerical experiments. For a slow time we use the notation $\theta=\varepsilon t$.

The data in the problem, the functions $F, G, \Lambda, \omega$ are supposed to be smooth infinitely differentiable functions and $2 \pi$-periodic in $\psi$. We consider the problem on constructing an asymptotic expansion as $\varepsilon \rightarrow 0$ for solutions $E(t ; \varepsilon), S(t ; \varepsilon)$, whose leading term in the asymptotics is determined by the $\operatorname{root} E=\mathcal{E}_{0}(\theta)$ of the equation $\Lambda(E, \theta)=0$. In what follows, the

L.A. Kalyakin, Capture and holding of Resonance far from Equilibrium.

(C)KALYAKIN L.A. 2018.

Submitted April 05, 2018. 
family of such solutions is called resonance solutions and they can be selected by posing a initial condition for the component $E$ in the vicinity of root:

$$
\left.E\right|_{t=0}=E_{\text {res }}+\sqrt{\varepsilon} r_{0} \quad \text { under the condition } \Lambda\left(E_{\text {res }}, 0\right)=0 .
$$

The set of admissible initial values for the second component $\left.S\right|_{t=0}=s_{0}$ and admissible perturbations $r_{0}$ form a so-called domain of capture into resonance; this will be provided below.

Since the structure of the equations is invariant with respect to the shift of the variables $E$, $S$, we can assume that $E_{\text {res }}=0$. We suppose that at the initial point the relations hold:

$$
\Lambda(0,0)=0,\left.\quad \partial_{E} \Lambda(E, 0)\right|_{E=0} \neq 0 .
$$

This condition ensures the existence of a simple root $E=\mathcal{E}_{0}(\theta)$ for the functional equation $\Lambda(E, \theta)=0$ in the segment $0 \leqslant \theta<\theta_{0}, \theta_{0}=$ const $\leqslant \infty$ with the property $\mathcal{E}_{0}(0)=0$. An issue being discussed in the present work is about the determining of the time interval $0 \leqslant \theta<\theta_{c}$, in which this root is the leading term of the asymptotics for the component $E(t ; \varepsilon)$ of the solution.

1.2. Survey of close problems. If the functions $F(E, S, \psi, \theta), G(E, S, \psi, \theta)$ are periodic in two variables $S, \psi$, differential equations (1) can be interpreted as a model of a nonlinear oscillator being under the action of an external perturbation periodic in $\psi$. The variable $\theta=\varepsilon t$ characterizes slow and in general non-periodic deformations of the system. As the perturbation is absent, that is, as $\varepsilon=0$, the quantity $E=$ const is constant and it is usually called energy of action. The function $S(t)$ is linear in $t$ and is interpreted as a phase or angle; $\Lambda(E, 0)$ is the oscillation frequency of the unperturbed oscillator. The dependence of this function on $E$ reflects a nonlinearity of the oscillator. Under such interpretation, the smoothness of the functions $F, G$ in the variable $E$ corresponds to the absence of the equilibrium for the unperturbed oscillator in the vicinity of the initial condition $E=0$. An analyse of the solutions describing the perturbation of the equilibrium differs from the constructions provided below and is not discussed here [1], 2].

Problems closed to ones considered here were studied a lot. The exhaustive results are known for weakly non-autonomous systems, as the fast phase $\psi$ is absent. In this case the leading terms in the asymptotics appropriate up to the time $t=\mathcal{O}\left(\varepsilon^{-1}\right)$ are determined by averaged equations [3], 4]. In particular, a weak deformation of the energy is found by the equation

$$
\frac{d E}{d \theta}=\frac{1}{2 \pi} \int_{0}^{2 \pi} F(E, S, \theta) d S .
$$

At that, an important condition is that the quantity $\Lambda$ should be non-zero; this quantity is interpreted as an eigenfrequency of the system. In such problems, the zero frequency is associated with the separatrix of the unperturbed oscillator, in the vicinity of which the averaging method turns out to be not applicable and the structure of the asymptotics becomes much more complex [5], [6], [7], [8].

If the dependence on $\psi$ is present, we can write out a similar equation, which is obtained by averaging with respect to two fast phases:

$$
\frac{d E}{d \theta}=\frac{1}{(2 \pi)^{2}} \int_{0}^{2 \pi} \int_{0}^{2 \pi} F(E, S, \psi, \theta) d S d \psi .
$$

But under condition (2), its solution can have no relation with the leading term in the asymptotics of the energy $E(t ; \varepsilon)$ at the times $t=\mathcal{O}\left(\varepsilon^{-1}\right)$. Problems arise in higher terms in the asymptotics and are due to the the presence of the resonances. In similar problems one should keep in mind that behind the averaging procedure, there is a formal constructing of oscillating higher order terms. Under condition (2) such construction in the class of the functions periodic in $S, \psi$ is impossible because of the resonances and the presence of the latter deforms essentially the structure of the asymptotic solution. 
For a model of oscillator with a given fast varying phase, it is advantageous to reduce the initial equations to a form, when the quantity $\Lambda(E, \theta)$ is the difference of eigenfrequence and forcing frequence and $S(t)$ is the difference of phases. As the frequencies are kept close for a long time, that is, $\Lambda(E, \theta) \approx 0$, this is interpreted as a capture into resonance. On such solutions the energy changes essentially and this is the aim of many applied problems [9], [10]. In the general case, the function $\Lambda$ can have a more complex structure with no relations with $\omega(\theta)$. The periodicity of $F$ and $G$ in $S$ is not assumed.

In order to describe the capture into resonance, the scale of slow time $\tau=\sqrt{\varepsilon} t$ is employed in asymptotic constructions, cf. [11, [12, [4]. The constructing of the leading term in the asymptotics of the phase $S=S_{0}(\tau)+\mathcal{O}\left(\varepsilon^{\frac{1}{2}}\right)$ is reduced to solving pendulum type equations for $S_{0}(\tau)$. In these constructions one finds a change of the energy $E(t ; \varepsilon)$ by a quantity of order $\mathcal{O}(\sqrt{\varepsilon})$. If the pumping frequency is constant $\omega=$ const, a more essential in order of $\varepsilon$ change of the energy is impossible: the system oscillates with the amplitude $\mathcal{O}(\sqrt{\varepsilon})$ periodically approaching the boundary of the resonance. Such phenomenon is interpreted as due to the nonlinearity. As the energy varies, the eigenfrequency changes, the resonance breaks and the growth of the energy stops.

However, if a given external frequency $\omega$ varies slowly in time, sometimes the resonance is kept at large time interval $0<t \leqslant \mathcal{O}\left(\varepsilon^{-1}\right)$ [13]. At that, the energy changes by a quantity of order one, $\mathcal{O}(1)$. Such situation is interpreted as an automatic tuning of the system for the external action, which called autoresonance or self-phasing, under which the eigenfrequence varies according the varying of the external frequency [9], [10, [1]. If the external frequency varies in the scale $\theta=\varepsilon t$, then the leading term of the asymptotics $E \approx \mathcal{E}_{0}(\theta)$ is found by the equation $\Lambda(E, \theta)=0$ corresponding to the condition of the capture into resonance [14]. This can make an impression that the resonance is captured on the entire segment $0<\theta<\theta_{0}$, in which the root $\mathcal{E}_{0}(\theta)$ is defined independently on the perturbations $F, G$. Nevertheless, such long capturing of the resonance is not always possible. The validity of resonance condition (2) at the initial moment and the presence of the resonance at times $\mathcal{O}\left(\varepsilon^{-\frac{1}{2}}\right)$ does not ensure its capture up to the time $\varepsilon^{-1} \cdot \theta_{0}$. The capture of the resonance depends on the initial perturbation and the structure of the functions $F(E, S, \psi, \theta), G(E, S, \psi, \theta)$ [15], [1]. The main issue discussed in the present work is to find a time interval $0<\theta<\theta_{c}$, in which the resonance is captured with description of the energy as the asymptotics $E=\mathcal{E}_{0}(\theta)+\mathcal{O}(\sqrt{\varepsilon}), \varepsilon \rightarrow 0$.

We should observe that the initial conditions can be written as

$$
\frac{d E}{d t}=\varepsilon F(E, S, \psi, I), \quad \frac{d S}{d t}=\Lambda(E, I)+\varepsilon G(E, S, \psi, I), \quad \frac{d I}{d t}=\varepsilon, \quad \frac{d \psi}{d t}=\omega(I)
$$

subject to the initial condition $\left.I\right|_{t=0}=0$. They can be considered as a particular case of a two-frequency system. Such problems for general two-frequency system under the absence of a slow time were studied via the approximation by leading terms [16], [17]. The justification of the asymptotics was also given in these works. In the present work, we suggests a rather simple construction of complete asymptotic solution for system (1) in a resonance zone based on the ideas of multi-scaled expansion. The main result is an equation, whose solution allows us to find the time of capturing the resonance.

1.3. Results. In the asymptotic construction we employ two slow times $\tau=\sqrt{\varepsilon} t$ and $\theta=\varepsilon t$. The leading terms of the asymptotics are constructed as

$$
E=\mathcal{E}_{0}(\theta)+\sqrt{\varepsilon} r(\tau, \theta ; \varepsilon) \mathcal{E}_{1}(\theta)+\mathcal{O}(\varepsilon), \quad S=s(\tau, \theta ; \varepsilon)+\mathcal{O}(\sqrt{\varepsilon}), \quad \varepsilon \rightarrow 0
$$

with the coefficient

$$
\mathcal{E}_{1}(\theta)=\frac{1}{\partial_{E} \Lambda\left(\mathcal{E}_{0}(\theta), \theta\right)}
$$


At the scale $\tau$, the problem is reduced to a model system of pendulum type, which is obtained by averaging in $\psi$ :

$$
\frac{d r}{d \tau}=f_{0}(s, \theta), \quad \frac{d s}{d \tau}=r, \quad(\theta=\sqrt{\varepsilon} \tau)
$$

Here

$$
f_{0}(s, \theta)=\langle F(E, s, \psi, \theta)\rangle \partial_{E} \Lambda(E, \theta)+\left.\partial_{\theta} \Lambda(E, \theta)\right|_{E=\mathcal{E}_{0}(\theta)}
$$

by small angle brackets $\langle\cdot\rangle$ we denote the mean of periodic functions in the variable $\psi$ :

$$
\langle f(\psi)\rangle=\frac{1}{2 \pi} \int_{0}^{2 \pi} f(\psi) d \psi
$$

If we consider $\theta$ as a frozen parameter, equations (4) can be integrated. Their solutions give asymptotics (3) at the stage of capture into resonance, that is, at time $t=\mathcal{O}\left(\varepsilon^{-\frac{1}{2}}\right)$. The variable $\theta$ in the form $\theta=\sqrt{\varepsilon} \tau$ is employed for describing the asymptotics at long time $t=\mathcal{O}\left(\varepsilon^{-1}\right)$. At that, one we have to analyze the problem on perturbation of system (4) and to take into consideration the correctors of order $\mathcal{O}(\sqrt{\varepsilon})$.

A necessary for capturing resonance is the presence of an oscillation domain $D_{o s}(\theta)$ in the phase plane $(r, s)$; this domain is formed by closed phase trajectories of frozen system (4), [17. This domain defines the set ${ }^{1}$ of initial data $\left(r_{0}, s_{0}\right) \in D_{0}=D_{o s}(0)$ ensuring the capture into resonance. The sufficient condition for the existence of oscillation domain is

Capture condition. As $0 \leqslant \theta<\theta_{0}$, frozen system (4) possesses a separatrix loop with one stable equilibrium inside. Then the oscillation domain $D_{\text {os }}(\theta)$ is the interior of the loop.

The asymptotics of resonance solutions (3) is described by means of periodic in $\tau$ functions $r(\tau, \theta ; \varepsilon), s(\tau, \theta ; \varepsilon)$, whose parameters slowly deform in the scale $\theta$. The moment of leaving the resonance is identified with the moment as the corresponding trajectory reachs the boundary of oscillation domain $\partial D_{o s}(\theta)$. It is convenient to formulate the conditions of capturing resonance as the inequality for the area $\Pi(\theta)$ enveloped by the trajectory and the area $\Pi_{o s}(\theta)$ of the domain $D_{o s}(\theta)$. The described approach is not new and is known for a long time. The main result of the present work is an equation for the evolution of the area $\Pi(\theta)$ on the base of an explicit presentation for the perturbation in the pendulum system. The general structure of the perturbation was pointed out in [16], [17]. The calculations given below allow us to write out the perturbation explicitly in terms of initial data and to proceed to the equation for the area in form (22). This equation can be a subject for further analysis and numerical experiments.

As an example, we provide a result for a particular case, when the equation for the area is simplified and we get a known statement [16].

Theorem 1. Suppose that the perturbation in original system (1) is Hamiltonian after averaging $\psi:\left\langle\partial_{E} F+\partial_{S} G\right\rangle=0$, and the function $\Lambda(E, \theta)=\Omega(E)-\omega(\theta)$ is the differene of the frequencies. Then a slow evolution of the area under the perturbed trajectory possesses the property

$$
\Pi(\theta) / \Omega^{\prime}\left(\mathcal{E}_{0}(\theta)\right)=\text { const }+\mathcal{O}(\sqrt{\varepsilon}), \quad \varepsilon \rightarrow 0 .
$$

The difference of formula (5) from corresponding relation in [16] is in the factor $1 / \Omega^{\prime}\left(\mathcal{E}_{0}(\theta)\right)$. This difference is not principle and is explained by another choice of the variable $r$ in asymptotic construction in deriving the pendulum system.

Corollary 1. Let the assumptions of Theorem 1 and capture condition hold. Then the resonance is captured at time until the inequalities

$$
\frac{\Omega^{\prime}\left(\mathcal{E}_{0}(\theta)\right)}{\Omega^{\prime}(0)}<\frac{\Pi_{o s}(\theta)}{\Pi(0)}, \quad \theta<\theta_{0}
$$

\footnotetext{
${ }^{1}$ capture domain
} 
are satisfied.

We note that the time of capturing resonance depends on the initial point of the trajectory $r_{0}, s_{0}$ by means of the initial value of the area $\Pi(0)$. It can be significantly smaller than the limiting value $\theta_{0}$.

\section{AsYmptotic CONSTRUCTION}

The base of the asymptotic construction is the passage from the unknown function $E(t ; \varepsilon)$ to a new variable $R(\tau ; \varepsilon), \tau=\sqrt{\varepsilon} t$ by using the equation

$$
\Lambda(E, \theta)=\sqrt{\varepsilon} R, \quad \theta=\sqrt{\varepsilon} \tau .
$$

Thanks to resonance condition (2), this equation possesses a smooth root $E=\mathcal{E}(\sqrt{\varepsilon} R, \theta)$ for all sufficiently small values of $\sqrt{\varepsilon} R$ and $\theta$. We can write out the Taylor expansion of this function uniform in $\theta$ :

$$
\mathcal{E}(\sqrt{\varepsilon} R, \theta)=\mathcal{E}_{0}(\theta)+\sqrt{\varepsilon} R \mathcal{E}_{1}(\theta)+\mathcal{O}\left(\varepsilon R^{2}\right), \quad \varepsilon R^{2} \rightarrow 0, \quad 0<\theta<\theta_{0} .
$$

The coefficients are expressed in terms of the derivatives, for instance,

$$
\mathcal{E}_{1}(\theta)=\frac{1}{\partial_{E} \Lambda(E, \theta)} \equiv-\frac{1}{\partial_{\theta} \Lambda(E, \theta)} \quad \text { as } \quad E=\mathcal{E}_{0}(\theta)
$$

We can treat formula (6) as an asymptotics as $\varepsilon \rightarrow 0$ uniform in $R$ on each compact set. In fact, this is an ansätz for the energy, in which we need to find a bounded function $R(\tau ; \varepsilon)$.

The equations for new variables $R(\tau ; \varepsilon), S(\tau ; \varepsilon)$ are of the the form

$$
\left.\frac{d R}{d \tau}=\mathcal{F}(E, S, \psi, \theta), \quad \frac{d S}{d \tau}=R+\sqrt{\varepsilon} G(E, S, \psi, \theta) \quad \text { as } \quad E=\mathcal{E}(\sqrt{\varepsilon} R, \theta)\right) .
$$

The right hands sides are compositions of functions of $(R, S, \tau)$ expressed in terms of the initial data, in particular,

$$
\mathcal{F}(E, S, \psi, \theta)=\partial_{E} \Lambda(E, \theta) F(E, S, \psi, \theta)+\partial_{\theta} \Lambda(E, \theta), \quad \frac{d \psi}{d \tau}=\frac{1}{\sqrt{\varepsilon}} \omega(\theta), \quad \theta=\sqrt{\varepsilon} \tau
$$

Equations (7) involve no explicit dependence on an intermediate variable $\tau$, they depend only on a slow time $\theta=\sqrt{\varepsilon} \tau$. The right hand sides depend periodically on a given fast phase $\psi$. It is convenient to extract explicitly the dependence of this variable in asymptotic constructions. At that, in the asymptotic solution we can separate the mean value of the sought functions from oscillating parts with zero mean.

We take ansätz as

$$
R(\tau, \varepsilon)=r(\tau, \theta ; \varepsilon)+\sqrt{\varepsilon} \mathcal{R}(r, s, \psi, \theta, \varepsilon), \quad S(\tau, \varepsilon)=s(\tau, \theta ; \varepsilon)+\sqrt{\varepsilon} \mathcal{S}(r, s, \psi, \theta, \varepsilon)
$$

supposing the zero mean: $\langle\mathcal{R}\rangle=0,\langle\mathcal{S}\rangle=0$. This ansätz contains three time scale: a fast scale is defined by the variable $\psi$, a slow scale is described by $\theta$ and an intermediate scale corresponds to $\tau$. We observe that the dependence on $\tau$ in the oscillating parts is involved only by help of mean values $r, s$. Under such approach we can separate the problem on determining oscillating parts $\mathcal{R}, \mathcal{S}$ from the problem on finding the mean values $r, s$. These ideas are contained in the known averaging method [3]. Equations for means can be obtained by averaging in $\psi$ :

$$
\begin{aligned}
& {\left[\frac{\partial}{\partial \tau}+\sqrt{\varepsilon} \frac{\partial}{\partial \theta}\right] r=\langle\mathcal{F}(E, s+\sqrt{\varepsilon} \mathcal{S}, \psi, \theta)\rangle,} \\
& {\left[\frac{\partial}{\partial \tau}+\sqrt{\varepsilon} \frac{\partial}{\partial \theta}\right] s=r+\sqrt{\varepsilon}\langle G(E, s+\sqrt{\varepsilon} \mathcal{S}, \psi, \theta)\rangle, \quad E=\mathcal{E}(\sqrt{\varepsilon} r+\varepsilon \mathcal{R}, \theta) .}
\end{aligned}
$$


These equations contain oscillating parts $\mathcal{R}, \mathcal{S}$ of the sought functions. The equations for these parts are written by taking into considerations the averaged ones:

$\begin{aligned} \omega(\theta) \frac{\partial \mathcal{R}}{\partial \psi}+\sqrt{\varepsilon} \frac{\partial \mathcal{R}}{\partial \theta}= & \mathcal{F}(E, s+\sqrt{\varepsilon} \mathcal{S}, \psi, \theta)-\langle\mathcal{F}(E, s+\sqrt{\varepsilon} \mathcal{S}, \psi, \theta)\rangle \\ & -\sqrt{\varepsilon} \partial_{r} \mathcal{R}\langle\mathcal{F}(E, s+\sqrt{\varepsilon} \mathcal{S}, \psi, \theta)\rangle-\sqrt{\varepsilon} \partial_{s} \mathcal{R}[r+\sqrt{\varepsilon}\langle G(E, s+\sqrt{\varepsilon} \mathcal{S}, \psi, \theta)\rangle], \\ \omega(\theta) \frac{\partial \mathcal{S}}{\partial \psi}+\sqrt{\varepsilon} \frac{\partial \mathcal{S}}{\partial \theta}= & \mathcal{R}+\sqrt{\varepsilon}[G(E, s+\sqrt{\varepsilon} \mathcal{S}, \psi, \theta)-\langle G(E, s+\sqrt{\varepsilon} \mathcal{S}, \psi, \theta)\rangle]\end{aligned}$

We note that in the latter relations the means $r, s$ are regarded as parameters, in which the dependence of $\tau$ is taken into consideration in accordance with the averaging construction. This allows us to separate the equations in asymptotic constructions.

The ansätz for oscillating parts of solutions is taken as the asymptotic series

$$
\begin{aligned}
& \mathcal{R}(t ; \varepsilon)=\mathcal{R}_{0}(s, \psi, \theta)+\sum_{k=1}^{\infty} \varepsilon^{\frac{k}{2}} \mathcal{R}_{k}(r, s, \psi, \theta), \\
& \mathcal{S}(t ; \varepsilon)=\mathcal{S}_{0}(s, \psi, \theta)+\sum_{k=1}^{\infty} \varepsilon^{\frac{k}{2}} \mathcal{S}_{k}(r, s, \psi, \theta) .
\end{aligned}
$$

The coefficients in 10 are determined uniquely in the class of functions with zero mean. For instance, at the first step we obtain equations implying the absence of the parameter $r$ in the leading term of the oscillating part of the solution:

$$
\omega(\theta) \frac{\partial \mathcal{R}_{0}}{\partial \psi}=\mathcal{F}_{0}(s, \psi, \theta)-f_{0}(s, \theta), \quad \omega(\theta) \frac{\partial \mathcal{S}_{0}}{\partial \psi}=\mathcal{R}_{0} .
$$

Here $\mathcal{F}_{0}=\mathcal{F}\left(\mathcal{E}_{0}(\theta), s, \psi, \theta\right), f_{0}=\left\langle\mathcal{F}_{0}\right\rangle$. The sought functions $\mathcal{R}_{k}, \mathcal{S}_{k}, k \geqslant 0$, are written in terms of the integrals with respect to $\psi$. They are determined by the recurrent formulae in the class of functions with zero mean.

\section{Averaged EQUATIOnS}

By structure of averaged equations (9) one can see easily that by constructing of oscillating part of the asymptotics in first $n$ terms one ensures the determining of means of order up to $\varepsilon^{\frac{n+1}{2}}$ in the scale $\tau$. For instance, the averaged equations with the first correctors taken into consideration are of the form

$$
\frac{d r}{d \tau}=f_{0}(s, \theta)+\sqrt{\varepsilon} f(r, s, \theta)+\mathcal{O}(\varepsilon), \quad \frac{d s}{d \tau}=r+\sqrt{\varepsilon} g(s, \theta)+\mathcal{O}(\varepsilon), \quad \theta=\sqrt{\varepsilon} \tau .
$$

The right hand sides are given by the formulae

$$
\begin{aligned}
& f_{0}(s, \theta)=\partial_{E} \Lambda(E, \theta)\langle F(E, s, \psi, \theta)\rangle+\left.\partial_{\theta} \Lambda(E, \theta)\right|_{E=\mathcal{E}_{0}(\theta)} ; \\
& f=\left\langle\mathcal{F}_{1}(r, s, \psi, \theta)\right\rangle, \quad g=\left\langle G\left(\mathcal{E}_{0}(\theta), s, \psi, \theta\right)\right\rangle, \\
& \mathcal{F}_{1}(r, s, \psi, \theta)=r \mathcal{E}_{1}(\theta) \partial_{E} \mathcal{F}\left(\mathcal{E}_{0}(\theta), s, \psi, \theta\right)+\mathcal{S}_{0}(s, \psi, \theta) \partial_{s} \mathcal{F}\left(\mathcal{E}_{0}(\theta), s, \psi, \theta\right) .
\end{aligned}
$$

For the leading terms of the asymptotics, as $\varepsilon=0$, we obtain pendulum kind equations (4). If the slow time $\theta$ is regarded as a frozen parameter, then equations (4) can be integrated. Their solution gives the leading terms of the asymptotics for the means $r=r_{0}(\tau, \theta)+\mathcal{O}(\sqrt{\varepsilon})$, $s=s_{0}(\tau, \theta)+\mathcal{O}(\sqrt{\varepsilon})$ valid at the time $0<\tau \leqslant \mathcal{O}(1)$. If we unfreeze the parameter $\theta$ by letting $\theta=\sqrt{\varepsilon} \tau$, the constructed functions $r_{0}(\tau, \theta), s_{0}(\tau, \theta)$ satisfy equations (4) up to $\mathcal{O}(\sqrt{\varepsilon})$. This error is kept for each smooth dependence on $\theta$. In order to obtain an approximation valid up to a time $0<\tau \leqslant \mathcal{O}\left(\varepsilon^{-1 / 2}\right)$, we need to find an appropriate dependence on $\theta$. The ways of solving such problems are well-known in the case when the frozen solution oscillates [4]. At that, in the perturbed equations, one has to take into consideration the correctors of order $\mathcal{O}(\sqrt{\varepsilon})$ given in 
(14). We note that (14) involves the function $\mathcal{S}_{0}(s, \psi, \theta)$, which is one of the components of the oscillating part. This term can be transformed by calculating the perturbation function $f$.

Lemma 1. The relation holds:

$f(r, s, \theta)=r \frac{1}{\partial_{E} \Lambda(E, \theta)} \partial_{E}\left[\langle F(E, s, \psi, \theta)\rangle \partial_{E} \Lambda(E, \theta)+\partial_{\theta} \Lambda(E, \theta)\right]_{E=\mathcal{E}_{0}(\theta)}-\frac{1}{2} \partial_{s}\left\langle\mathcal{R}_{0}^{2}(s, \psi, \theta)\right\rangle$.

Proof. The first term in (15) is obtained by (14) once we take into consideration (8) and $\mathcal{E}_{1}=1 / \partial_{E} \Omega\left(\mathcal{E}_{0}(\theta), \theta\right)$.

The second term in (15) is implied (14) thanks to equations (11). In order to see that, we differentiate in $s$ the first identity in (11):

$$
\partial_{s} \mathcal{F}\left(\mathcal{E}_{0}(\theta), s, \psi, \theta\right)=\omega(\theta) \partial_{\psi} \partial_{s} \mathcal{R}_{0}+\partial_{s} f_{0}(s, \theta) .
$$

Since the mean in $\psi$ of the oscillating part $\left\langle\mathcal{S}_{0}(s, \psi, \theta)\right\rangle=0$ is zero, in averaging of the latter identity we get:

$$
\left\langle\mathcal{S}_{0}(s, \psi, \theta) \partial_{s} \mathcal{F}\left(\mathcal{E}_{0}(\theta), s, \psi, \theta\right)\right\rangle=\omega(\theta)\left\langle\mathcal{S}_{0}(s, \psi, \theta) \partial_{\psi} \partial_{s} \mathcal{R}_{0}\right\rangle .
$$

We recall that angle brackets stand for the integral with respect to $\psi$ over the period. Integrating by parts in the integral and employing the second identity in (11), we obtain:

$$
\left\langle\mathcal{S}_{0} \partial_{S} \mathcal{F}\right\rangle=-\omega(\theta)\left\langle\partial_{\psi} \mathcal{S}_{0} \partial_{s} \mathcal{R}_{0}\right\rangle=-\left\langle\mathcal{R}_{0} \partial_{s} \mathcal{R}_{0}\right\rangle=-\frac{1}{2} \partial_{s}\left\langle\mathcal{R}_{0}^{2}\right\rangle
$$

This leads us to relation (15).

\section{Perturbation of Pendulum Kind system}

We consider a model system of two equations perturbed by small terms:

$$
\frac{d r}{d \tau}=f_{0}(s, \theta)+\sqrt{\varepsilon} f(r, s, \theta) \quad 0<\varepsilon \ll 1, \quad \frac{d s}{d \tau}=r+\sqrt{\varepsilon} g(r, s, \theta), \quad \theta=\sqrt{\varepsilon} \tau .
$$

A smooth function $f_{0}(s, \theta)$ is assumed to be $2 \pi$-periodic in $s$. Its mean value $\hat{f}_{0}(\theta)=\left\langle f_{0}(s, \theta)\right\rangle$, not necessary being zero, is a smooth function in $\theta$. The construction of the asymptotics for the solution as $\varepsilon \rightarrow 0$ on the large time interval $0<\tau \leqslant \mathcal{O}\left(\varepsilon^{-\frac{1}{2}}\right)$ is based on the results for a frozen unperturbed system (4):

$$
\frac{d r}{d \tau}=f_{0}(s, \theta), \quad \frac{d s}{d \tau}=r
$$

in which $\theta$ is regarded as a parameter. Such problems were studied long time ago for general systems [4]. The main idea is to reduce the equation to the variables action-angle followed by the averaging. To have a complete presentation, we provide the details of a known approach for particular system (16).

The frozen system possesses a first integral. In order to write it out, it is convenient to introduce a potential $u(s, \theta)$ by the integral

$$
u(s, \theta)=-\int^{s} f_{0}(s, \theta) d s=-s \cdot \hat{f}_{0}(\theta)+\tilde{u}(s, \theta)
$$

fixing the integration constant, for instance, by the zero mean of the oscillating part $\tilde{u}(s, \theta)$. The potential involves a non-periodic term of form $s \cdot \hat{f}_{0}(\theta)$. The first integral representing the Hamiltonian of the system is written as

$$
\frac{1}{2} r^{2}+u(s, \theta)=h .
$$


Asymptotic constructions for the solution to the perturbed system are introduced in the domain of the phase plane $(r, s) \in D_{o s} \subseteq \mathbb{R}^{2}$ filled by the closed trajectories of the frozen system 1 . In the general case this domain $D_{o s}(\theta)$ depends on the parameter $\theta$ and this corresponds to its slow deformation in time. Closed frozen trajectories are associated with the values $h$ in some segment $H_{0}(\theta)<h<H_{1}(\theta)$, whose end-points generally speaking depend on $\theta$. The corresponding solutions to the frozen system, two periodic functions $r_{0}\left(\tau+\tau_{0}, h, \theta\right)$, $s_{0}\left(\tau+\tau_{0}, h, \theta\right)$ depend on two integration constants $\tau_{0}, h$ and on the parameters $\theta$. The period $T(h, \theta)$ and the frequency $\nu(h, \theta)=2 \pi / T$ of the solutions also depend on the parameters $h, \theta$ and are calculated via the integral over the trajectory. For further purposes it is convenient to introduce $2 \pi$-periodic functions by the formulae

$$
\rho(\varphi, h, \theta)=r_{0}\left(\frac{\varphi}{\nu}, h, \theta\right), \quad \sigma(\varphi, h, \theta)=s_{0}\left(\frac{\varphi}{\nu}, h, \theta\right) .
$$

The introduced functions satisfy the equations

$$
\nu \frac{\partial \rho}{\partial \varphi}=-\partial_{\sigma} u(\sigma, \theta), \quad \nu \frac{\partial \sigma}{\partial \varphi}=\rho .
$$

We differentiate the energy identity $\rho^{2}+2 u(\sigma, \theta)=2 h$ in $h$ and $\theta$ and in view of the equations this gives two relations, which can be written in terms of the determinants

$$
\nu\left|\begin{array}{ll}
\partial_{\varphi} \rho & \partial_{h} \rho \\
\partial_{\varphi} \sigma & \partial_{h} \sigma
\end{array}\right|=-1, \quad \nu\left|\begin{array}{cc}
\partial_{\varphi} \rho & \partial_{\theta} \rho \\
\partial_{\varphi} \sigma & \partial_{\theta} \sigma
\end{array}\right|=\partial_{\theta} u(\sigma, \theta)
$$

A constructed in this way pair of functions is employed in a change of variables in the perturbed equations

$$
r(\tau ; \varepsilon)=\rho(\varphi, h, \theta), \quad s(\tau ; \varepsilon)=\sigma(\varphi, h, \theta), \quad \theta=\sqrt{\varepsilon} \tau .
$$

New unknown functions are $h(\tau ; \varepsilon), \varphi(\tau ; \varepsilon)$. In the unperturbed equations such change describes the passage to the variable of action-angle type. The perturbed equations for $r, s$ are transformed to equations for $h, \varphi$. In fact, such approach corresponds to the variations of constants:

$$
\frac{d h}{d \tau}=\sqrt{\varepsilon}\left(\partial_{\theta} u(\sigma, \theta)-\nu\left|\begin{array}{cc}
\partial_{\varphi} \rho & f \\
\partial_{\varphi} \sigma & g
\end{array}\right|\right), \quad \frac{d \varphi}{d \tau}=\nu+\sqrt{\varepsilon} \nu\left(\left|\begin{array}{cc}
\partial_{\theta} \rho & \partial_{h} \rho \\
\partial_{\theta} \sigma & \partial_{h} \sigma
\end{array}\right|-\left|\begin{array}{ll}
f & \partial_{h} \rho \\
g & \partial_{h} \sigma
\end{array}\right|\right) .
$$

Here $\rho(\varphi, h, \theta), \sigma(\varphi, h, \theta), \nu(h, \theta)$ are known functions of the variables $\varphi, h$ and $\theta=\sqrt{\varepsilon} \tau$.

The obtained equations are similar to original system (1) with the only difference that here the frequency $\nu(h, \theta)$ does not vanish. In this case the asymptotic construction for the solution $h, \varphi$ as $\varepsilon \rightarrow 0$ is well-known [4]. The leading term of the asymptotics valid for large time

$$
h(\tau ; \varepsilon)=h_{0}(\theta)+\mathcal{O}(\sqrt{\varepsilon}), \quad \varepsilon \rightarrow 0, \quad \theta=\sqrt{\varepsilon} \tau, \quad 0<\tau \leqslant \mathcal{O}\left(\varepsilon^{-\frac{1}{2}}\right)
$$

is determined by the averaged equation

$$
\frac{d h_{0}}{d \theta}=Z\left(h_{0}, \theta\right)
$$

The right hand side is given by the expression

$$
Z(h, \theta)=\nu\left\langle f(\rho, \sigma, \theta) \partial_{\varphi} \sigma-g(\rho, \sigma, \theta) \partial_{\varphi} \rho\right\rangle+\left\langle\partial_{\theta} u(\sigma, \theta)\right\rangle, \rho, \sigma(\varphi, h, \theta),
$$

where the averaging is taken with respect to the phase $\varphi$ fast in the intermediate scale $\tau$.

The asymptotics for the fast variable $\varphi=\varphi(\tau ; \varepsilon)$ can be obtained by integrating the second equation in (18).

Such construction gives an asymptotic for solution $r(\tau ; \varepsilon), s(\tau ; \varepsilon)$ in terms of periodic functions (solutions to the frozen system), whose parameters slowly deform in the scale $\theta$. The

\footnotetext{
${ }^{1}$ if such domain exists.
} 
time interval, where this asymptotics is valid is limited by the values of $\theta_{c}$, for which the approximate unfrozen trajectory approaches the boundary of the oscillation domain $\partial D_{o s}(\theta)$. If the boundary is a separatrix loop, then as a trajectory approaches the boundary, the frequency $\nu(h, \theta)$ tends to zero and the approximation for $h$ obtained by the averaging method become invalid for time close to $\theta_{c},[5]$.

\section{Double AVERAGing}

The leading term of the asymptotics for solutions to the original problem valid for the time up to $t=\mathcal{O}\left(\varepsilon^{-1}\right)$ is determined by averaged equations (12). These equations are perturbation of pendulum type equations. The asymptotics is written in terms of the solution $\rho(\varphi, h, \theta)$, $\sigma(\varphi, h, \theta)$ to the frozen system rescaled to the period $2 \pi$. In order to such asymptotics

$$
r(\tau, \theta ; \varepsilon)=\rho(\varphi, h, \theta)+\mathcal{O}(\sqrt{\varepsilon}), \quad s(\tau, \theta ; \varepsilon)=\sigma(\varphi, h, \theta)+\mathcal{O}(\sqrt{\varepsilon})
$$

be valid up to long time $\tau=\mathcal{O}\left(\varepsilon^{-\frac{1}{2}}\right)$, we need to find a slow deformation of the parameter $h=h_{0}(\theta)+\mathcal{O}(\sqrt{\varepsilon})$. The presence of $h_{0}(\theta)$ ensures the validity of the approximation by the solution to the pendulum system periodic in $\varphi$ in the interval $0 \leqslant \theta<\theta_{c}$ until the frozen trajectory is located inside the oscillation domain. The asymptotics for the phase $\varphi=\varphi(\tau, \varepsilon)$, $\varepsilon \rightarrow 0$, is found by the second equation in (18). The leading term $h_{0}(\theta)$ ensures the error of order constant for the phase at long time $\tau=\mathcal{O}\left(\varepsilon^{-\frac{1}{2}}\right)$. To specify the asymptotics for $\varphi$, one has to calculate the correctors of order $\mathcal{O}(\sqrt{\varepsilon})$ for the parameter $h$; this is a usual situation while perturbing nonlinear oscillating systems. To find the bound $\theta_{c}$ and to estimate the error in ansätz (3) such specification of the phase is not needed.

In the considered case the perturbed system of pendulum type with the first corrected taken into consideration is of form (16). Perturbations functions $f, g$ are determined by formulae (13), (14) involving the averaging in the fast phase $\psi$. Calculation of the deformation of $h_{0}(\theta)$ is reduced to resolving equations $(19)$, in which the right hand side (20) involves the averaging in $\varphi$ in the intermediate scale $\tau$. This is how a double averaging arises.

Lemma 2. The right hand side of averaged equation (19) is expressed in terms of the original data by means of the averaging integrals in $\psi, \varphi$ as

$$
Z(h, \theta)=\partial_{\theta}\langle u(\sigma, \theta)\rangle+\left\langle\rho^{2}\left\langle\partial_{E} F(E, \sigma, \psi, \theta)+\partial_{\sigma} G(E, \sigma, \psi, \theta)\right\rangle\right\rangle+\left.\left\langle\rho^{2}\right\rangle \partial_{E} \frac{\partial_{\theta} \Lambda(E, \theta)}{\partial_{E} \Lambda(E, \theta)}\right|_{E=\mathcal{E}_{0}(\theta)} .
$$

Here $\rho(\varphi, h, \theta), \sigma(\varphi, h, \theta)$ are periodic solutions to a frozen system of pendulum type with the potential $u(\sigma, \theta)=\int f_{0}(\sigma, \theta) d \sigma$.

Proof. Let us calculate the mean value integrals involved in the right hand side of equation (19). The function $f$ defined in (14) is of the form

$f(\rho, \sigma, \theta)=\rho \frac{1}{\partial_{E} \Lambda\left(\mathcal{E}_{0}(\theta), \theta\right)} \partial_{E}\left[\langle F(E, \sigma, \psi, \theta)\rangle \partial_{E} \Lambda(E, \theta)+\partial_{\theta} \Lambda(E, \theta)\right]_{E=\mathcal{E}_{0}(\theta)}-\frac{1}{2} \partial_{\sigma}\left\langle\mathcal{R}_{0}^{2}(\sigma, \psi, \theta)\right\rangle$.

The latter term in calculating the mean $\left\langle f \partial_{\varphi} \sigma\right\rangle$ vanishes:

$$
\left\langle\partial_{\varphi} \sigma \partial_{\sigma}\left\langle\mathcal{R}_{0}^{2}(\sigma, \psi, \theta)\right\rangle\right\rangle=\left\langle\partial_{\varphi}\left\langle\mathcal{R}_{0}^{2}\left(S_{0}, \psi, \theta\right)\right\rangle\right\rangle=0
$$

independently of the properties of the oscillating in $\psi$ function $\mathcal{R}_{0}$. Then in view of the equation $\nu \partial_{\varphi} \sigma=\rho$, the remaining part of the double averaging $\nu\left\langle f \partial_{\varphi} \sigma\right\rangle$ involves the square of the function $\rho(\varphi, h, \theta)$ :

$$
\nu\left\langle f \partial_{\varphi} \sigma\right\rangle=\left.\frac{1}{\partial_{E} \Lambda(E)} \partial_{E}\left[\left\langle\rho^{2}\langle F(E, \sigma, \psi, \theta)\rangle\right\rangle \partial_{E} \Lambda(E, \theta)+\left\langle\rho^{2}\right\rangle \partial_{\theta} \Lambda(E, \theta)\right]\right|_{E=\mathcal{E}_{0}(\theta)} .
$$


In this expression, the derivative in $E$ is moved inside the brackets and the averaged equation of the form

$$
\langle F(E, s, \psi, \theta)\rangle=\frac{\nu \partial_{\varphi} \rho-\partial_{\theta} \Lambda(E, \theta)}{\partial_{E} \Lambda(E, \theta)}
$$

is taken into consideration. Since the mean of the derivative $\left\langle\partial_{\varphi} \rho\right\rangle=0$ vanishes, there remains the expression

$$
\nu\left\langle f \partial_{\varphi} \sigma\right\rangle=\left\langle\rho^{2}\left\langle\partial_{E} F(E, \sigma, \psi, \theta)\right\rangle\right\rangle+\left.\left\langle\rho^{2}\right\rangle \frac{1}{\partial_{E} \Lambda(E)} \partial_{E}\left[\frac{\partial_{\theta} \Lambda(E, \theta)}{\partial_{E} \Lambda(E, \theta)}\right]\right|_{E=\mathcal{E}_{0}(\theta)}
$$

While calculating the mean $\left\langle g \partial_{\varphi} \rho\right\rangle$, we employ the representation $g=\left\langle G\left(\mathcal{E}_{0}, \sigma(\varphi, H, \theta), \psi, \theta\right)\right\rangle$. In the double averaging the integral in $\varphi$ is calculated by parts and the identity $\nu \partial_{\varphi} \sigma=\rho$ is used:

$$
-\nu\left\langle g \partial_{\varphi} \rho\right\rangle=\nu\left\langle\partial_{\varphi} \sigma\left\langle\partial_{\sigma} G\left(\mathcal{E}_{0}, \sigma(\varphi, H, \theta), \psi, \theta\right)\right\rangle \rho\right\rangle=\left\langle\left\langle\partial_{\sigma} G\left(\mathcal{E}_{0}, \sigma, \psi, \theta\right)\right\rangle \rho^{2}\right\rangle .
$$

Thus, expression (20) becomes (21). The proof is complete.

In particular cases, expression (21) can be simplified.

Corollary 2. Let the function $\Lambda(E, \theta)$ be the difference of the frequencies

$$
\Lambda(E, \theta)=\Omega(E)-\omega(\theta) .
$$

Then the potential is given by the formula

$$
u(s, \theta)=-\Omega^{\prime}\left(\mathcal{E}_{0}(\theta)\right) \int^{s}\left\langle F\left(\mathcal{E}_{0}(\theta), s, \psi, \theta\right)\right\rangle d s+s \cdot \omega^{\prime}(\theta),
$$

and the right hand of the averaged equation reads as

$$
Z\left(h_{0}, \theta\right)=\partial_{\theta}\langle u(\sigma, \theta)\rangle+\left\langle\rho^{2}\left\langle\partial_{E} F(E, \sigma, \psi, \theta)+\partial_{\sigma} G(E, \sigma, \psi, \theta)\right\rangle\right\rangle+\frac{\mathcal{E}_{0}^{\prime}(\theta) \Omega^{\prime \prime}\left(\mathcal{E}_{0}(\theta)\right)}{\Omega^{\prime}\left(\mathcal{E}_{0}(\theta)\right)}\left\langle\rho^{2}\right\rangle .
$$

To prove the corollary, one should take into consideration

$$
\mathcal{E}_{0}^{\prime}(\theta)=-\left.\frac{\partial_{\theta} \Lambda(E, \theta)}{\partial_{E} \Lambda(E, \theta)}\right|_{E=\mathcal{E}_{0}(\theta)}=\frac{\omega^{\prime}(\theta)}{\Omega^{\prime}\left(\mathcal{E}_{0}(\theta)\right)}
$$

implied by $\Lambda\left(\mathcal{E}_{0}(\theta), \theta\right) \equiv 0$.

Corollary 3. Let the averaged in $\psi$ perturbation be Hamiltonian:

$$
\left\langle\partial_{E} F+\partial_{S} G\right\rangle=0
$$

and the function $\Lambda(E, \theta)$ is the difference of the frequencies $\Lambda(E, \theta)=\Omega(E)-\omega(\theta)$. Then the right hand side of the averaged equation reads as

$$
Z\left(h_{0}, \theta\right)=\partial_{\theta}\langle u(\sigma, \theta)\rangle+\frac{\mathcal{E}_{0}^{\prime}(\theta) \Omega^{\prime \prime}\left(\mathcal{E}_{0}(\theta)\right)}{\Omega^{\prime}\left(\mathcal{E}_{0}(\theta)\right)}\left\langle\rho^{2}\right\rangle .
$$

Here $\rho\left(\varphi, h_{0}, \theta\right), \sigma\left(\varphi, h_{0}, \theta\right)$ are periodic solutions to the frozen system and the averaging is taken with respect to $\varphi$. 


\section{AREA DEFORMATion}

Given an equation obtained by the double averaging, it is difficult to extract from it the information on the moment of resonance breaking as the trajectory approaches the boundary of oscillating domain. However, this equation can be reduced to a more convenient form by excluding the potential.

In the phase plane we consider an area $\Pi$ of the domain enveloped by the frozen trajectory $r=\rho(\varphi, h, \theta), s=\sigma(\varphi, h, \theta)$. The relations hold:

$$
\Pi=\oint \rho d \sigma=\int_{0}^{2 \pi} \rho(\varphi, h, \theta) \partial_{\varphi} \sigma(\varphi, h, \theta) d \varphi=2 \pi\left\langle\rho \partial_{\varphi} \sigma\right\rangle=\frac{2 \pi}{\nu}\left\langle\rho^{2}\right\rangle .
$$

It is convenient to employ this quantity instead of $h$ for parametrization of the family of periodic solutions. Its advantage is a simpler equation for the slow deformation.

Theorem 2. The evolution of the area under a slowly deforming trajectory of the averaged system of pendulum type (12) in the leading term of the asymptotics $\Pi=\Pi_{0}(\theta)+\mathcal{O}(\sqrt{\varepsilon})$ is described by the equation

$$
\frac{d \Pi_{0}}{d \theta}=\frac{2 \pi}{\nu}\left\langle\rho^{2}\left\langle\partial_{E} F(E, \sigma, \psi, \theta)+\partial_{\sigma} G(E, \sigma, \psi, \theta)\right\rangle\right\rangle+\left.\Pi_{0} \partial_{E} \frac{\partial_{\theta} \Lambda(E, \theta)}{\partial_{E} \Lambda(E, \theta)}\right|_{E=\mathcal{E}_{0}(\theta)} .
$$

Here $\rho\left(\varphi, h_{0}, \theta\right), \sigma\left(\varphi, h_{0}, \theta\right)$ are periodic solutions of the frozen system, $\nu\left(h_{0}, \theta\right)$ is a frequency, and the averaging is taken with respect to $\varphi$.

Remark. In the general case the equation for $\Pi_{0}(\theta)$ is non-trivial since the area depends on the trajectory, that is, on the parameter $h$.

Proof of Theorem 2. The dependence of $\Pi(\theta)$ on the slow time $\theta$ is involved both explicitly and via the parameter $h=h(\theta ; \varepsilon)$. This is why, integrating by parts in one of the integrals, for the derivative we obtain the relation

$$
\frac{1}{2 \pi} \frac{d \Pi}{d \theta}=\left\langle\left[\partial_{h} \rho \partial_{\varphi} \sigma-\partial_{\varphi} \rho \partial_{h} \sigma\right]\right\rangle \frac{d h}{d \theta}+\left\langle\left[\partial_{\theta} \rho \partial_{\varphi} \sigma-\partial_{\varphi} \rho \partial_{\theta} \sigma\right]\right\rangle .
$$

In view of identity 17 ) and the averaged equation $(19)$, for the leading terms of the asymptotics

$$
\Pi=\Pi_{0}(\theta)+\mathcal{O}(\sqrt{\varepsilon}), \quad h=h_{0}(\theta)+\mathcal{O}(\sqrt{\varepsilon})
$$

we obtain:

$$
\frac{1}{2 \pi} \frac{d \Pi_{0}}{d \theta}=\frac{1}{\nu} \frac{d h_{0}}{d \theta}-\frac{1}{\nu} \partial_{\theta} u(\sigma, \theta)
$$

Taking into consideration equation 19$)$ for $h_{0}(\theta)$ and the expression for the right hand side in (21), we get

$$
\frac{\nu}{2 \pi} \frac{d \Pi_{0}}{d \theta}=\left\langle\rho^{2}\left\langle\partial_{E} F(E, \sigma, \psi, \theta)+\partial_{\sigma} G(E, \sigma, \psi, \theta)\right\rangle\right\rangle+\left.\left\langle\rho^{2}\right\rangle \partial_{E} \frac{\partial_{\theta} \Lambda(E, \theta)}{\partial_{E} \Lambda(E, \theta)}\right|_{E=\mathcal{E}_{0}(\theta)} .
$$

Having in mind the relation of the mean $\left\langle\rho^{2}\right\rangle$ with the area, we arrive at $(22)$. The proof is complete.

In view of equation 22 we see that as $\partial_{E} F+\partial_{S} G=0$, a parametric deformation of the potential $u(s, \theta)$ and Hamiltonian perturbation have no influence for the leading term of the asymptotics of the area. In the Hamiltonian case the equation for the area is simplified significantly. 
Corollary 4. Assume that the perturbation in the original system is Hamiltonian after the averaging in $\psi:\left\langle\partial_{E} F+\partial_{S} G\right\rangle=0$. Then the slow evolution of the area is described by the relation

$$
\frac{d \Pi_{0}}{d \theta}=\left.\Pi_{0} \cdot \partial_{E} \frac{\partial_{\theta} \Lambda(E, \theta)}{\partial_{E} \Lambda(E, \theta)}\right|_{E=\mathcal{E}_{0}(\theta)} .
$$

Equation (23) is obviously integrated. The simplest expression is obtained in the particular case described in Theorem 1 .

Corollary 5. Assume that the perturbation in the original system is Hamiltonian after the averaging in $\psi$ and the function $\Lambda(E, \theta)=\Omega(E)-\omega(\theta)$ is a difference of frequencies. Then the slow evolution of the area is described by the formula $\Pi_{0}(\theta)=\Pi(0) \Omega^{\prime}\left(\mathcal{E}_{0}(\theta)\right) / \Omega^{\prime}\left(\mathcal{E}_{0}(0)\right)$, which agrees with Theorem 1 .

Proof. In the considered case the deformation equation reads as

$$
\frac{d \Pi_{0}}{d \theta}=\frac{\mathcal{E}_{0}^{\prime}(\theta) \Omega^{\prime \prime}\left(\mathcal{E}_{0}(\theta)\right)}{\Omega^{\prime}\left(\mathcal{E}_{0}(\theta)\right)} \Pi_{0} .
$$

Formula (5) provides its solution.

The obtained formula for the area implies immediately Corollary 1. We observe that the results for this particular case (Hamiltonian perturbations) correspond to the known results [16], [17].

In a non-Hamiltonian case the equation for the area can be sometimes integrated. For instance, in the model taking into consideration the dissipation as follows:

$$
\left\langle\partial_{E} F(E, \sigma, \psi, \theta)+\partial_{\sigma} G(E, \sigma, \psi, \theta)\right\rangle=\beta(\theta)<0,
$$

the function $\Pi_{0}(\theta)$ decays exponentially in $\theta$.

\section{Conclusion}

We analyze a problem on the time of existence of a resonance for a nonlinear oscillating system with a small (of order $\varepsilon \ll 1$ ) resonance perturbation. In the asymptotic approximation, the problem is reduced to a model system of pendulum type. Under this approximation, the time of the resonance breaking $t_{c} \approx \varepsilon^{-1}$ is identified with the moment, when a slowly deforming trajectory approaches the boundary of the oscillation domain. For the area under the frozen trajectory we write out deformation equation $(22)$. Its solution allows us to write a functional equation for the moment of the resonance breaking. The simplest case corresponds to Hamiltonian perturbations.

\section{BIBLIOGRAPHY}

1. L.A. Kalyakin. Asymptotic analysis of autoresonance models // Uspekhi Matem. Nauk. 63:5, 3-72. [Russ. Math. Surv. 63:5, 791-857 (2008).]

2. A.I. Neishtadt, A.A. Vasiliev and A.V. Artemyev. Capture into resonance and escape from it in a forced nonlinear pendulum // Regul. Chaot. Dynam. 18:6, 691-701 (2013).

3. N.N. Bogolyubov, Yu.A. Mitropol'sky. Asymptotic methods in the theory of non-linear oscillations. Nauka, Moscow (1974). [Gordon and Breach, New York (1961)].

4. V.I. Arnold, V.V. Kozlov, A.I. Neishtadt. Mathematical aspects of classical and celestial mechanics. VINITI, Moscow (1985). [Springer, Berlin (1997).]

5. A.I.Neishtadt. Passage through a separatrix in a resonance problem with a slowly-varying paramete // Prikl. Matem. Mekh. 39:4, 621-632 (1975). [J. Appl. Math. Mech. 39:4, 594-605 (1975).]

6. O.M. Kiselev and S.G. Glebov. An asymptotic solution slowly crossing the separatrix near a saddle-centre bifurcation point // Nonlinearity. 16:1, 327-362 (2003). 
7. A. Neishtadt, A. Vasilliev Phase change between separatrix crossing in slow-fast Hamiltonian systems // Nonlinearity. 18:3, 1393-1406 (2005).

8. O.M. Kiselev. Oscillations near a separatrix in the Duffing equation// Trudy IMM UrO RAN. 18:2, 141-153 (2012). [Proc. Stekl. Inst. Math. 281, Suppl. 1, 82-94 (2013).]

9. K.S. Golovanivsky. Autoresonant acceleration of electrons at nonlinear ECR in a magnetic field which is smoothly growing in time // Physica Scripta. 22:2, 126-133 (1980).

10. K.S. Golovanivsky. The gyromagnetic autoresonance // IEEE Trans. Plasma Sci. 11:1, 28-35 (1983).

11. G.M. Zaslavskii, R.Z. Sagdeev. Introduction to nonlinear physics. From the pendulum to turbulence and chaos. Nauka, Moscow (1977). (in Russian).

12. M.I. Rabinovich, D.I. Trubetskov. Introduction into oscillation and wave theory. Regul. Chaot. Dyn., Izhevsk (2000). (in Russian).

13. B.V. Chirikov. The passage of a nonlinear oscillating system through resonance // Dokl. Akad. Nauk SSSR. 125:5, 1015-1018 (1959). [Sov. Phys. Dokl. 4, 390-394 (1959).]

14. L.A. Kalyakin. Averaging in the autoresonance model // Matem. Zamet. 73:3, 449-452 (2003). [Math. Notes. 73:3, 414-418 (2003).]

15. A.P. Itin, A.I. Neishtadt, A.A. Vasiliev. Capture into resonance in dynamics of a charged partice in magnetic field and electrostatic wave // Physica D. 141:4, 281-296 (2000).

16. A.I. Neishtadt. Capture into resonance and scattering on resonances in two-frequency systems // Trudy MIAN. 250, 198-218 (2005). [Proc. Steklov Inst. Math. 250, 183-203 (2005).]

17. A.I. Neishtadt. Averaging, passage through resonances, and capture into resonance in twofrequency systems // Uspekhi Matem. Nauk. 69:5, 3-80 (2014). [Russ. Math. Surv. 69:5, 771-843 (2014).]

Leonid Anatolevich Kalyakin,

Institute of Mathematics,

Ufa Federal Research Center, RAS,

Chernyshevsky str. 112,

450008, Ufa, Russia,

E-mail: klenru@mail.ru 